\title{
Osteogenic differentiation of human adipose-derived stem cells: comparison of two different inductive media
}

\author{
Laura de Girolamo ${ }^{1,2}$, Matteo F. Sartori ${ }^{1}$, Walter Albisetti ${ }^{3}$ and Anna T. Brini ${ }^{1 *}$ \\ ${ }^{1}$ Medical Pharmacology Department, Faculty of Medicine, University of Milan, Italy \\ ${ }^{2}$ Gateozzi Orthopaedic Institute, Milan, Italy \\ ${ }^{3}$ Orthopaedic and Traumatology Institute, Faculty of Medicine, University of Milan, Italy
}

\begin{abstract}
Human mesenchymal stem cells (MSCs) have the potential to differentiate into cells of connective tissue lineages, including bone, cartilage, fat, muscle and also neurons. In our study we have examined the phenotypic profile of human adipose tissue-derived stem cells (hASCs) and compared different osteogenic-inductive media to assess hASC differentiation. Cells were enzymatically isolated from adipose tissues derived by liposuction from several adult human donors, purified and then expanded in culture. We obtained an abundant yield of hASCs with a constant proliferative trend, a doubling time of about $68 \mathrm{~h}$ and a mild variable clonogenic capacity. At passage 4, hASCs expressed MSC-related cell surface antigens (CD13, CD105, CD54, CD90, CD44), and subsequently hASCs were induced to differentiate into the osteogenic lineage for at least 3 weeks of culture in two distinct media, OM1 and OM2, differing in dexamethasone and ascorbic acid concentrations. Osteogenic differentiation of OM1- and OM2-cultured cells was assessed by evaluating cell morphology, osteopontin expression, alkaline phosphatase activity and calcium deposition. OM2 medium showed a higher osteogenic potential than OM1, as assessed by increased levels of calcium deposition, alkaline phospatase activity and osteopontin expression in comparison with OM1-differentiated cells. We conclude that hASCs efficiently differentiate into osteogenic lineage, particularly when cultured in inductive medium supplemented with $10 \mathrm{~nm}$ dexamethasone and $150 \mu \mathrm{M}$ ascorbic acid. Copyright $\odot 2007$ John Wiley \& Sons, Ltd.
\end{abstract}

Received 13 December 2006; Revised 30 January 2007; Accepted 7 February 2007

Keywords adult stem cells; adipose-derived stem cells; skeletal tissue regeneration; osteogenic differentiation; dexamethasone; ascorbic acid

\section{Introduction}

Although bone marrow represents the most common source of mesenchymal stem cells (MSCs), other tissues, such as periostium, muscle, synovial membrane and adipose tissue, also contains MSCs (Le Blanc and Ringden, 2006; Hattori et al., 2004). In particular, adipose tissue is a very abundant and precious MSC source, due to the ease of withdrawing cells from it and its high abundance.

\footnotetext{
*Correspondence to: Anna T. Brini, Medical Pharmacology Department, Faculty of Medicine, University of Milan, Italy. E-mail: anna.brini@unimi.it
}

Human adipose-derived stem cells (hASCs) have been shown to possess multilineage potential to differentiate into bone, cartilage, fat, muscle, endothelial and neural cells (Zuk et al, 2001; De Ugarte et al., 2003; Dudas et al., 2006; Dragoo et al., 2003; Planat-Benard et al., 2004; Choi et al., 2006). We here analyse the differentiation capacity of hASCs cultured in two different osteogenic-inductive media.

hASCs were obtained by collagenase digestion of lipoaspirate from 20 donors, aged $21-60$ years, under informed consent (Zuk et al., 2002; Erickson et al., 2002; Awad et al., 2004); $80 \%$ were females and $20 \%$ males; $60 \%$ of the donors were aged $20-40$ years and the other $40 \%$ 41-60 years. No body mass index (BMI) data were 
collected. Cells were cultured in DMEM supplemented with $10 \% \mathrm{FBS}, 50 \mathrm{U} / \mathrm{ml}$ penicillin, $50 \mu \mathrm{g} / \mathrm{ml}$ streptomycin and $2 \mathrm{~mm}$ L-glutamine (control medium, CTRL). From $1 \mathrm{ml}$ raw lipoaspirate we separated about $5 \times 10^{5}$ cells, which theoretically would be able to generate $10^{9}$ MSCs in 1 month. Their proliferation rate was quite constant, with a doubling time of about $68 \mathrm{~h}$. No significant difference in growth rate was observed between cells derived from different lipoaspirates. Colony formation of the hASC population was determined by CFU-F assay. Their clonogenic potential was variable, with a minimal percentage of $3 \%$ to a maximum of $12 \%$ of clonogenic cells (data not shown).

At passage 4, when the cell population reached a fibroblast-like shape and they appeared to form a homogenous population with similar size and granularity, their phenotypic profile was investigated by cytofluorimetric analysis using a FACSCalibur System (Becton-Dickinson, NJ, USA).

More than $90 \%$ of hASC cells expressed CD13, CD90 (Thy-1) and CD105/SH2 (Endoglin); about 90\% were $\mathrm{CD}_{4}{ }^{+}$, around $80 \% \mathrm{CD}^{2} 9^{+}$, about 75\% CD54 (ICAM- 1$)^{+}$ and $30-85 \%$ expressed CD49d, whereas there was very low expression of CD14 (around 1-4\%) and no expression of CD45, CD71 and CD106 was detected, confirming previous published data (De Ugarte et al., 2003; Lee et al., 2004). After surface marker characterization, hASCs were then differentiated towards the osteogenic lineage by culturing them at a density of 15000 cells $/ \mathrm{cm}^{2}$ in two different media: OM1 (CTRL medium supplemented with $10 \mathrm{~mm} \beta$-glycerol phosphate, $100 \mathrm{~nm}$ dexamethasone, $50 \mu \mathrm{M}$ ascorbic acid-2-phosphate); and OM2 (CTRL plus $10 \mathrm{~mm} \beta$-glycerol phosphate, $10 \mathrm{nM}$ dexamethasone and $150 \mu \mathrm{M}$ ascorbic acid-2-phosphate). The cells were cultured for at least 28 days, changing the medium twice weekly, and their differentiation capacity compared.

The differences in osteogenic differentiation potential in cells cultured in OM1 and OM2 media were assessed by evaluating cell morphology, osteopontin expression, alkaline phosphatase activity and calcium deposition. Pronounced morphological differences between undifferentiated and OM1-cultured hASCs were highlighted by haematoxylin and eosin (H\&E) staining (Figure 1A; Shi et al., 2005). Two weeks of osteogenic differentiation in both media did not modify CD13, CD 90 and CD14 expression, determined by FACS analysis (data not shown), whereas we observed a significant upregulation of osteopontin (OPN) expression. A slightly more evident OPN expression in OM2-cultured cells was detectable (Figure 1B), as shown by immunofluorescence. FACS analysis on permeabilized hASCs confirmed this result and allowed the increase of OPN expression in OM1grown hASCs of about 50\% towards undifferentiated cells to be quantified (data not shown).

In addition, after 21 days, OM2 medium induced higher levels of extracellular matrix formation than in OM1culture conditions, as shown by alizarin red $\mathrm{S}$ staining, a calcium-specific marker (Figure 2A). Quantification analysis determined by optical density measurements, after cetylpyridinium chloride extraction as described by Halvorsen et al. (2001), showed a significant increase in calcium deposition of about 24 times for cells differentiated in OM2 medium compared with cells maintained in OM1 medium, and about 32 times compared with CTRL cells. However, no significant increase of calcium deposition in OM1-differentiated cells was detected compared with CTRL cells. Additional analysis at different time points indicates that calcium
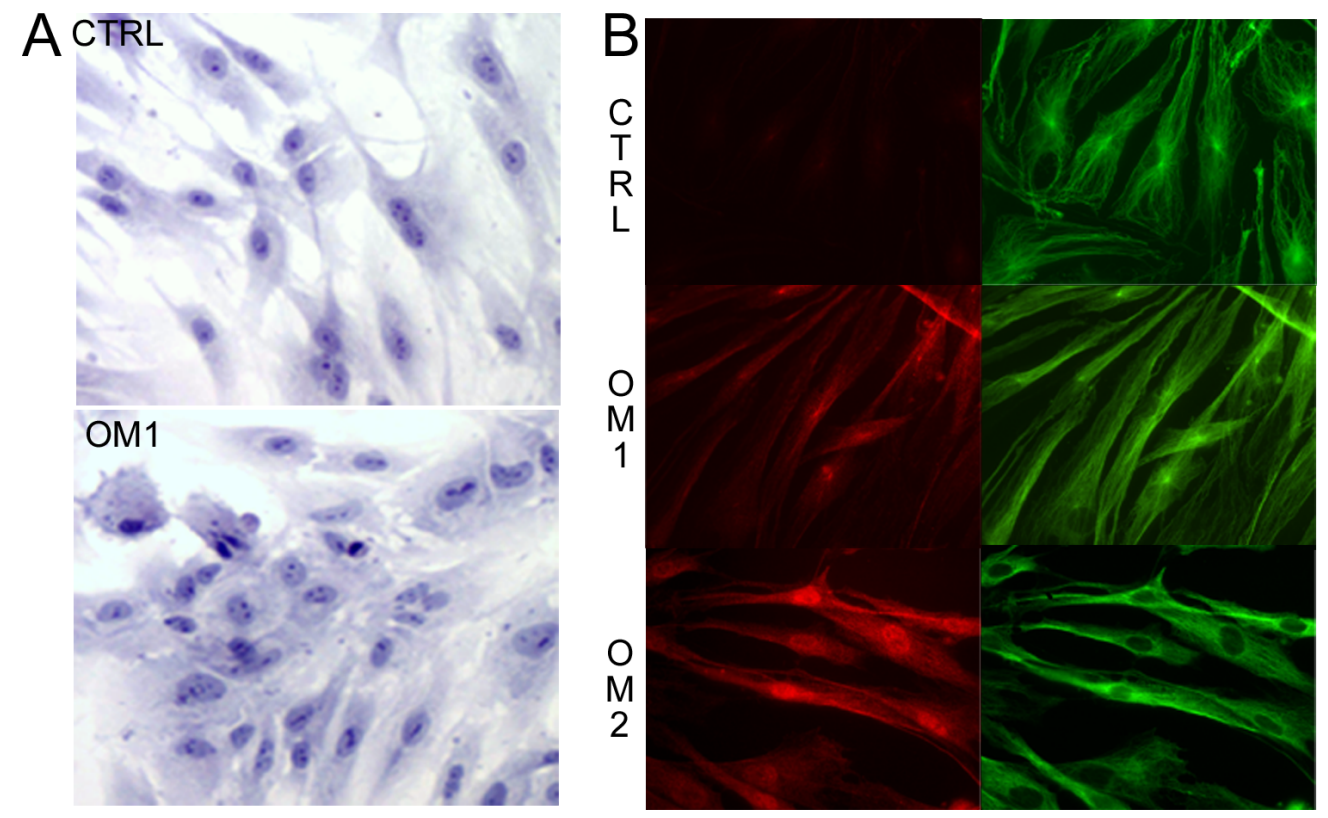

Figure 1. Undifferentiated (CTRL) and differentiated hASCs (OM1 and OM2). Cell morphology as shown by (A) H\&E staining (CTRL and OM1-cultured hASCs) and (B) osteopontin protein expression. In the immunofluorescence experiments, the cells were stained with either rabbit anti-osteopontin or mouse anti- $\alpha$-tubulin primary antibodies, subsequently revealed by TRITC- and FITC-conjugated secondary antibodies, respectively 


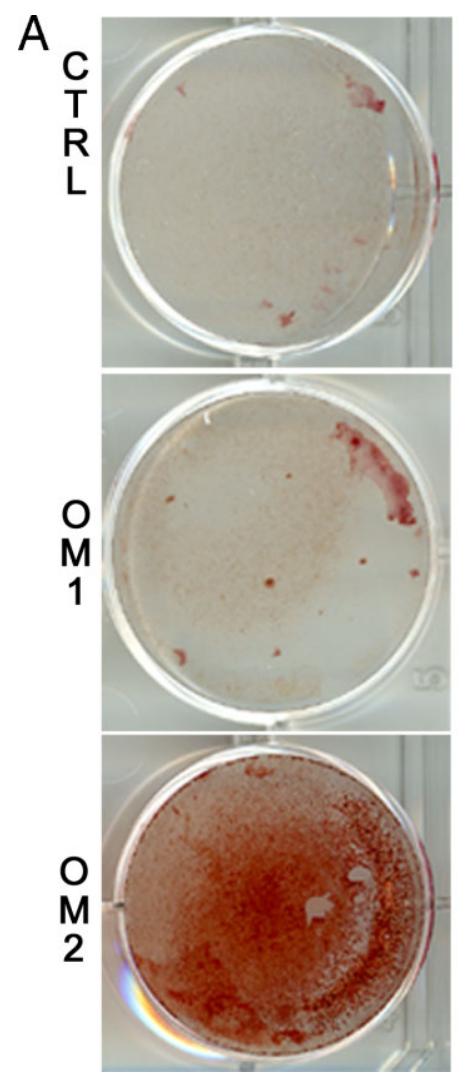

$\mathrm{B}$

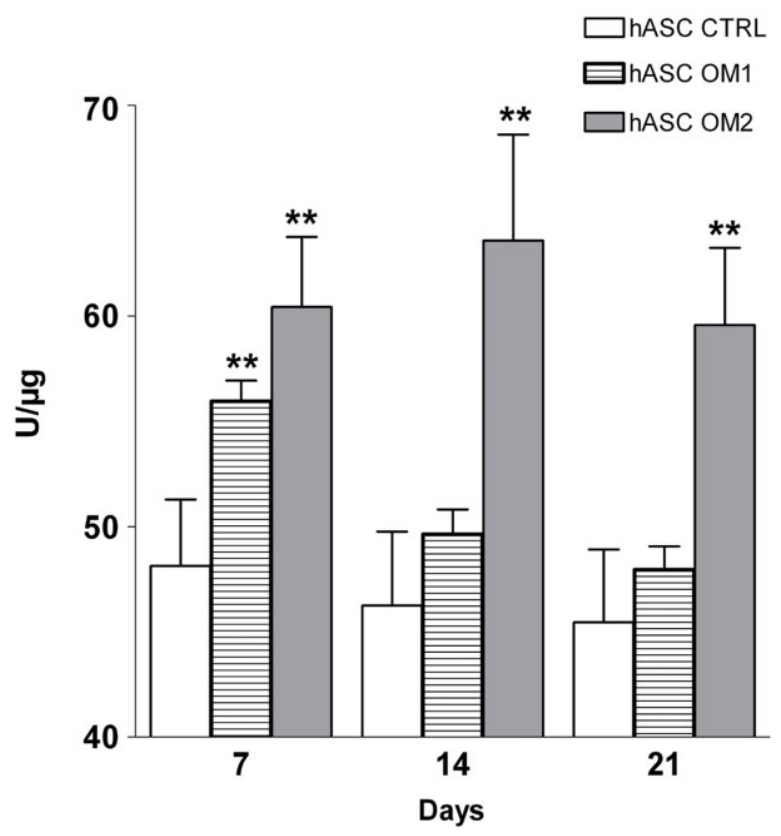

Figure 2. Osteogenic differentiation marker. (A) Calcium deposition of OM1- and OM2-differentiated hASCs determined by alizarin red S staining. (B) Alkaline phosphatase modulation in CTRL and OM1- and OM2-grown cells at different times of culture. Enzymatic activity levels are standardized on total protein concentration and expressed as U/ $\mu \mathrm{g}$. Bars represent mean \pm SD of triplicate determinations of at least three independent experiments

deposition was detectable by 10 days of differentiation and increased in the presence of $10 \mathrm{~nm}$ cholecalcipherol (data not shown).

The osteogenic potential of OM1- and OM2-cultured hASCs was confirmed by studies on alkaline phosphatase (ALP) kinetic activity at days 7, 14 and 21. After 7 days of differentiation, a significant increase of alkaline phosphatase activity (ALP), was detected in both OM1and OM2-cultured cells compared with undifferentiated cells. The observed upregulation was maintained for 3 weeks when the cells were cultured in OM2, whereas the ALP activities of hASCs grown in OM1 were not significantly different from those of undifferentiated cells at 14 and 21 days (Figure 2B). The addition of $10 \mathrm{~nm}$ cholecalcipherol to OM2 further increased either ALP activity and calcium deposition in differentiated hASCs (data not shown).

Western blot experiments are in progress to evaluate a panel of specific proteins whose expression will be modulated during differentiation.

hASCs are also able to generate cells of the chondrogenic lineage when the cells are cultured in a pellet system in the presence of Insulin, Transferrin, Selenium (ITS) and TGF $\beta 1$, and of the adipogenic lineage as determined by Alcian blue and Oil red-O staining, respectively (data not shown, paper in preparation). We conclude that subcutaneous adipose tissue is a good source of MSCs. hASCs are able to proliferate in undifferentiated conditions, maintaining their MSC phenotype. In the presence of appropriate stimuli they differentiated into osteoblasts, and low dexamethasone concentration and increased ascorbic acid concentration in particular seem to be faster and better osteogenic inducers (Kim et al., 2003). Further studies on the adherence properties and filling capacities on several scaffolds of OM2-cultured hASCs are in progress.

\section{References}

Awad HA, Wickham MQ, et al. 2004; Chondrogenic differentiation of adipose-derived adult stem cells in agarose, alginate, and gelatin scaffolds. Biomateriale 25(16): 3211-3222.

Choi YS, Cha SM, et al. 2006; Adipogenic differentiation of adipose tissue-derived adult stem cells in nude mouse. Biochem Biophys Res Commun 345: 631-637.

De Ugarte DA, Morizono K, et al. 2003; Comparison of multi-lineage cells from human adipose tissue and bone marrow. Cell Tissues Organs 174(3): 101-109.

Dragoo JL, Choi JY, et al. 2003; Bone induction by BMP-2 transduced stem cells derived from human fat. J Orthop Res 21(4): 622-629.

Dudas JR, Marra KG, et al. 2006; The osteogenic potential of adipose-derived stem cells for the repair of rabbit calvarial defects. Ann Plast Surg 56(5): 543-548.

Erickson GR, Gimble JM, et al. 2002; Chondrogenic potential of adipose tissue-derived stromal cells in vitro and in vivo. Biochem Biophys Res Commun 290(2): 763-769.

Halvorsen YC, Franklin D, et al. 2001; Extracellular matrix mineralization and osteoblast gene expression by human adipose tissue-derived stromal cells. Tissue Eng 7(6): 729-741. 
Hattori H, Sato M, et al. 2005; Osteogenic potential of human adipose tissue-derived stromal cells as an alternative stem cell source. Cells Tissues Organs 178: 2-12.

Kim H, Kim HW, Suh H. 2003; Sustained release of ascorbate2-phosphate and dexamethasone from porous PLGA scaffolds for bone tissue engineering using mesenchymal stem cells. Biomaterials 24: 4671-4679.

Le Blanc K, Ringden O. 2006; Mesenchymal stem cells: properties and role in clinical bone marrow transplantation. Curr Opin Immunol 18: 586-591.

Lee RH, Kim B, et al. 2004; Characterization and expression analysis of mesenchymal stem cells from human bone marrow and adipose tissue. Cell Physiol Biochem 14(4-6): 311-324.
Planat-Benard V, Silvestre JS, et al. 2004; Plasticity of human adipose lineage cell toward endothelial cells. Circulation 109: 656-663.

Shi YY, Nacamuli RP, et al. 2005; The osteogenic potential of adipose-derived mesenchymal cells is maintained with aging. Plast Reconstr Surg 116(6): 1686-1696.

Zuk PA, Zhu M, et al. 2001; Multilineage cells from human adipose tissue: implications for cell-based therapies. Tissue Eng 7(2): 211-228.

Zuk PA, Zhu M, et al. 2002; Human adipose tissue is a source of multipotent stem cells. Mol Biol Cell 13(12): 4279-4295. 